\title{
Mid-infrared Type-II InAs/InAsSb Quantum Wells Integrated on Silicon
}

\author{
E. Delli, ${ }^{1, a)}$ P. D. Hodgson, ${ }^{2}$ M. Bentley, ${ }^{1}$ E. Repiso, ${ }^{2}$ A. P. Craig, ${ }^{2}$ Q. \\ Lu, ${ }^{2}$ R. Beanland, ${ }^{3}$ A. R. J. Marshall, ${ }^{2}$ A. Krier, ${ }^{2}$ and P. J. \\ Carrington $^{1, b)}$ \\ ${ }^{1}$ Department of Engineering, Lancaster University, Bailrigg, Lancaster, LA1 4YW, UK \\ ${ }^{2}$ Department of Physics, Lancaster University, Bailrigg, Lancaster, LA1 4YB, UK \\ ${ }^{3}$ Department of Physics, University of Warwick, Gibber Hill Rd, Coventry, CV4 7AL, UK
}

\begin{abstract}
Direct integration of III-V semiconductor light sources on silicon is an essential step towards the development of portable, on-chip infrared sensor systems. Driven by the presence of characteristic molecular fingerprints in the mid-infrared spectral region, such systems may have a wide range of applications in infrared imaging, gas sensing and medical diagnostics. This paper reports on the integration of an InAs virtual substrate and high crystalline quality InAs/InAsSb multi-quantum wells on Si using a three-stage InAs/GaSb/Si buffer layer. It is shown that the InAs/GaSb interface demonstrates a strong dislocation filtering effect. A series of strained AlSb/InAs dislocation filter superlattices were also used, resulting in a low surface dislocation density of approximately $4 \times 10^{7}$ $\mathrm{cm}^{-2}$. The InAs/InAsSb wells exhibited strong photoluminescence signal at elevated temperatures. Analysis of these results indicate that radiative recombination is the dominant recombination mechanism, making this structure promising for fabricating MIR Si-based sensor systems.
\end{abstract}

The presence of fundamental vibration absorption bands of several gaseous species in the 2 to $12 \mu \mathrm{m}$ mid-infrared (MIR) electromagnetic spectral region presents high technological potential for a wide range of applications, including absorption spectroscopy, environmental monitoring, chemical sensing and medical diagnostics. MIR silicon ( $\mathrm{Si}$ ) photonics has attracted great interest due to its potential to realize lab-on-chip optoelectronic systems. Si wafers have numerous advantageous properties, such as their large area, improved robustness and low cost. ${ }^{1}$ Fabrication

\footnotetext{
a) Author to whom correspondence should be addressed. Electronic mail: evdelli@physics.auth.gr

b) Author to whom correspondence should be addressed. Electronic mail: p.carrington@lancaster.ac.uk
} 
compatibility with mature CMOS industrial technology is an additional benefit. In recent years, the development of Si-based MIR passive electronic components such as waveguides and resonators enhanced the prospect of low-cost, fully integrated photonic circuits ${ }^{2}$. However, fabrication of high-performance group IV-based light emitting and detection devices is still challenging, mainly due to the indirect band gap of Si. In contrast, many III-V compound semiconductors have direct bandgaps and robust photonic properties ${ }^{3}$. Therefore, integration of III-Vs onto Si or silicon-on-insulator (SOI) platforms is seen as the most promising approach for the fabrication of group-IV compatible light sources. Presently, III-V photonic components are connected on Si platforms mainly using bonding technologies. Even though such fabrication processes have been used to develop hybrid III-V-on-Si infrared sensors and laser arrays ${ }^{4}$, large scale application is still challenging due to the complexity and increased production cost of these techniques. Thus, direct heteroepitaxial growth of III-Vs on Si is considered one of the most promising methods for producing dense optoelectronic integrated circuits at high volume. However, even this approach faces major physical challenges due to material dissimilarities. The polar/non-polar character of the III-V/Si interface, large lattice mismatch and thermal expansion coefficient differences introduce various crystal defects into the epilayer, such as antiphase domains (APDs) and threading dislocations (TDs). These defects can strongly degrade the performance of optical devices and therefore high-quality epitaxial growth is required.

InAs and InAs-based compounds are one of the most promising candidates for infrared photonic and electronic applications due to their high electron mobilities ${ }^{5}$ and tunable, directbandgap, which spans across the infrared spectral region. Furthermore, type-II InAs-based structures, such as InAs/InAsSb quantum wells (QWs) and superlattices (SLs) ${ }^{6}$, provide remarkable flexibility to engineer the band structure, enabling suppression of Auger recombination whilst demonstrating defect tolerance due to the placement of defect states above the conduction band edge ${ }^{7}$. However, deposition of InAs on a planar (100) Si surface occurs by the formation of large InAs islands during the growth of the first monolayers, resulting in a high density of stacking faults $^{8}$ and APDs ${ }^{9}$. Early work on the epitaxial growth of InAs on Si focussed on the use of a GaAs intermediate layer ${ }^{10,11,12}$. However the large lattice mismatch between InAs and GaAs ( 7\%) resulted in a highly defective interface with a large density of threading dislocations ${ }^{10}$. More recent work has investigated the use of $\mathrm{GaP} / \mathrm{Si}^{13}$ and $\mathrm{Ge}$-on-Si combined with an AlInAs graded buffer 
layer ${ }^{14}$ to accommodate the large lattice mismatch between InAs and Si. This led to the subsequent demonstration of a room temperature InAs p-i-n photodetector. InAs-based SLs ${ }^{15}$ and QCLs ${ }^{16,17}$ have also been demonstrated on Si using a GaSb bulk buffer layer to help accommodate the high misfit strain. High performance MIR InAs/InAsSb SL photodetectors ${ }^{18}$ on Si have also recently been developed using a GaSb buffer layer grown by molecular beam epitaxy (MBE). This consisted of an AlSb interfacial misfit array and a two-temperature-step GaSb buffer layer which suppressed the vertical propagation of threading dislocations. The work reported in this paper describes an alternative method to integrate high crystalline quality InAs onto Si using this $\mathrm{GaSb} / \mathrm{Si}$ buffer as an intermediate transition layer. Additionally, MIR type-II InAs/InAsSb multiquantum wells (MQWs) were heteroepitaxially grown on top of a three-stage InAs/GaSb/Si buffer.

Samples were grown using a solid source Veeco GENxplor MBE system on Si wafers with a $4^{\circ}$ miscut towards the [0-11] direction. The Si substrates were prepared for growth using an in-situ thermal cleaning procedure at temperatures up to $1000{ }^{\circ} \mathrm{C}$. Initially a thin (17 monolayer) AlSb nucleation layer was deposited, followed by a $2 \mu \mathrm{m}$ thick GaSb buffer layer using a two-step growth temperature technique $\left(490{ }^{\circ} \mathrm{C}\right.$ and $\left.515^{\circ} \mathrm{C}\right)$. This provides a smooth, APD free GaSb epilayer on Si with a lattice constant close to InAs. Details of the Si wafer cleaning technique, the growth of the GaSb buffer layer and the surface characterization have been described previously ${ }^{18,19}$. Next, an InAs-based buffer was grown to provide a hybrid InAs-on-Si platform. This consisted of a thick $1 \mu \mathrm{m}$ InAs layer and a series of AlSb/InAs dislocation filter superlattices (DFSLs) as shown in Figure 1(a). Each superlattice period consisted of five repeats of AlSb (10 $\mathrm{nm}) / \mathrm{InAs}(10 \mathrm{~nm})$, with a total of five periods, each separated by a $500 \mathrm{~nm}$ thick InAs spacer. Strain at the interface between the layers of the DFSLs encourages bending of the TDs, increasing the likelihood that two will intercept or annihilate, leading to a reduction of the TDs density in subsequent layers. The DFSLs were grown using a low growth temperature of $450{ }^{\circ} \mathrm{C}$, to minimize the exchange of group-V anions at the InAs/AlSb interface, and at growth rates of $1 \mathrm{ML} / \mathrm{sec}$ and $0.31 \mathrm{ML} / \mathrm{sec}$ for the InAs and AlSb layers respectively. The bulk InAs layers $(1 \mu \mathrm{m}$ thick InAs layer and spacer layers) were grown using a temperature of $483{ }^{\circ} \mathrm{C}$. Ramping of the substrate temperature between each DFSL set had the added benefit of annealing the sample, promoting migration of TDs and improving the material quality. Finally, type-II InAs/InAsSb MQWs for photoluminescence (PL) were grown consisting of ten periods of $\mathrm{InAs}_{\mathrm{N}} \mathrm{InAs} \mathrm{As}_{0.95} \mathrm{Sb}_{0.05}$ with each 
period having a thickness of $31 \mathrm{~nm}(\mathrm{InAs}=23 \mathrm{~nm}, \mathrm{InAsSb}=8 \mathrm{~nm})$. An optimized shutter sequence consisted of $20 \mathrm{sec}$ of As flux incident the InAsSb surface prior to the growth of InAs layers in order to minimize the interface composition gradient as described previously ${ }^{20}$.

(a)

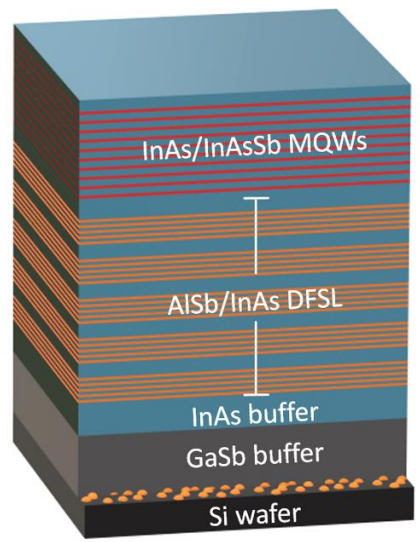

(b)

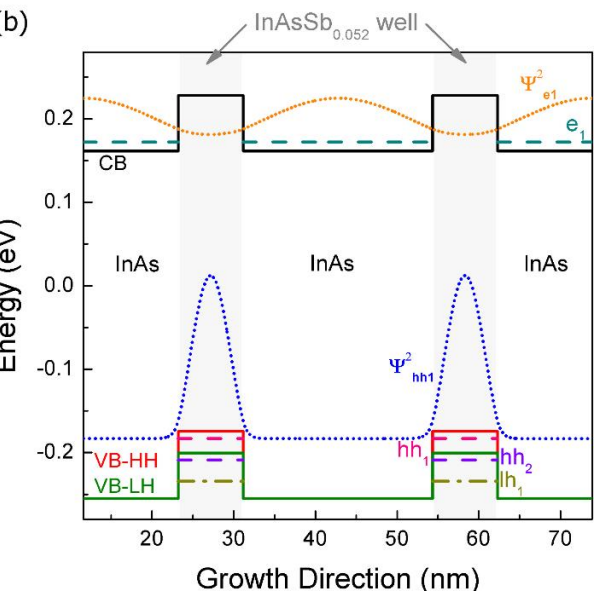

FIG. 1. (a) Schematic of the InAs/InAsSb MQW structure grown on GaSb/Si using the InAsbased DFSL buffer structure. The thickness of the InAs-based buffer structure was $3.6 \mu \mathrm{m}$. (b) Calculated band structure of the InAs/InAsSb MQWs at $15 \mathrm{~K}$ showing the first electron band, the first two heavy hole bands, the first light hole band and the wavefunctions $\left(\Psi_{\mathrm{i}}^{2}\right)$. 
A JEOL 2100 transmission electron microscope (TEM) operating at $200 \mathrm{kV}$ was used to collect the TEM images. AFM surface images were obtained using a Multimode Atomic Force Microscope in tapping mode and a Nanoscope 8 controller. The AFM scanning area was $10 \mu \mathrm{m} x$ $10 \mu \mathrm{m}$. Electron channeling contrast images (ECCI) were collected using a Zeiss Gemini SEM with a solid-state backscatter detector operating at 20. An incident beam orientation at the intersection of the 220 and 2-20 Kikuchi bands was used to accurately measure the surface defect density. Finally, photoluminescence (PL) characterization was carried out using a Bruker Vertex 70 Fourier Transform Infrared (FTIR) spectrometer, a $77 \mathrm{~K} \mathrm{InSb}$ photodiode detector and an OptistatDN-V2 cryostat. High-resolution X-ray diffraction (XRD) was carried out using a Bede QC200 diffractometer. Modeling of the diffraction pattern was used to determine the $\mathrm{Sb}$ composition and layer thicknesses of the InAs/InAsSb MQWs. The resulting values were then used to calculate the band structure and band offsets of the wells using Nextnano software ${ }^{18,21}$ (the bulk value of InAs, $6.0583 \AA$, was used in the simulations). Figure 1(b) shows the calculated typeII band alignment of the InAs/InAsSb MQWs at low temperature (15 K) along with the electron, heavy hole and light hole levels. The transition energies between the electron ground state to the first and second heavy hole states were calculated as $355 \mathrm{meV}$ and $381 \mathrm{meV}$ respectively.

Figures 2(a) and (c) together show the complete structure from the Si substrate to the MQWs. Figure 2(b) shows the $\mathrm{GaSb} / \mathrm{Si}$ interface in detail with a high resolution lattice image and geometric phase analysis using Strain $++^{22}$. The $12.3 \%$ misfit strain is completely accommodated by an array of edge dislocations, which appears perfect at this small field of view but as shown in Fig. 2(a) actually gives rise to a significant density $\left(\sim 10^{9} \mathrm{~cm}^{-2}\right)$ of threading dislocations. Most of these dislocations are deflected by the $0.62 \%$ strain at the InAs/GaSb interface, which helpfully leads to dislocation interactions and annihilation of threading dislocations ${ }^{23}$. This threading dislocation reduction was further improved by the inclusion of five dislocation filter superlattices as shown in Fig. 2(c). A small density of microtwins $\left(<10^{6} \mathrm{~cm}^{-2}\right)$ nucleated at the InAs:GaSb interface (not shown). As discussed by Okumura et $\mathrm{al}^{24}$, growth of InAs on $\mathrm{GaSb}$ proceeds with simultaneous formation of two dimensional islands alongside a step-flow mode. Generation of planar defects may be due to the formation of faceted InAs islands ${ }^{25}$. The hybrid InAs-on-Si structure was also free of thermal cracks. AFM surface characterization of the sample revealed a root mean square (rms) surface roughness of $3 \mathrm{~nm}$ (Fig. 2(e)). This value is approximately 3 times lower than InAs layers grown on $\mathrm{GaP} / \mathrm{Si}$ and 1.4 times lower than layers 

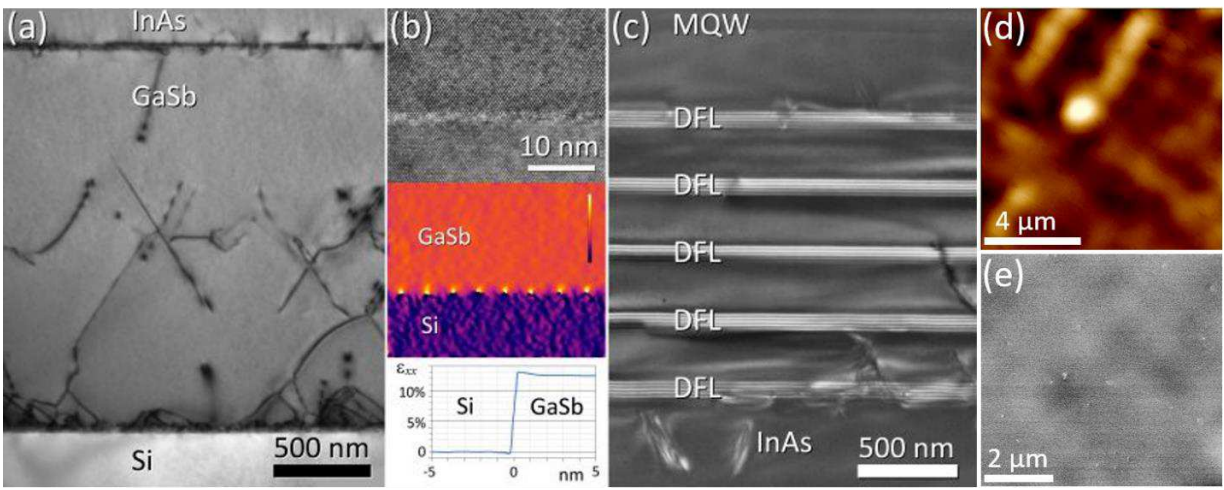

FIG. 2. (a) Cross-sectional bright field $\mathbf{g}=220 \mathrm{TEM}$ image of the InAs/GaSb buffer, showing threading dislocations originating at the $\mathrm{GaSb} / \mathrm{Si}$ interface. (b) High resolution image and geometric phase analysis of the $\mathrm{GaSb} / \mathrm{Si}$ interface showing a regular array of Lomer dislocations and complete accommodation of $12.3 \%$ strain. (c) Dark field $\mathbf{g}=002$ image showing the five sets of AlSb/InAs dislocation filters with the MQW at the top of the structure. (d) AFM image (area: $10 \times 10 \mu \mathrm{m}$ ) and (e) ECCI image of the top surface showing threading dislocations with a density of $\sim 4 \times 10^{7} \mathrm{~cm}^{-2}$.

grown on $\mathrm{Ge} / \mathrm{Si}^{26}$. The final threading dislocation density at the MQW layers and the top surface was approximately $4 \times 10^{7} \mathrm{~cm}^{-2}$, as shown in the ECCI image of Figure 2(d). This is significantly lower compared to that reported previously for $\mathrm{GaSb} / \mathrm{Si}$ buffer layers ${ }^{18}$, confirming the strong filtering effect of the InAs-based DFSL buffer structure.

The high-resolution XRD pattern of the InAs/InAsSb MQWs PL sample is shown in Figure 3(a). Many well-defined and intense peaks can be observed, indicating the excellent crystalline quality of the structure. These peaks are attributed to the wells, the DFSLs and the bulk InAs, GaSb and Si materials. A simulation of the XRD pattern suggested a MQW period thickness of $31.2 \mathrm{~nm}$ and an Sb content $5.2 \%$ in the InAsSb layers, which is in excellent agreement with the target values ( $\mathrm{Sb}$ : $5 \%$, period thickness: $31 \mathrm{~nm}$ ). The XRD data suggests that the individual thickness of the layers is $23.2 \mathrm{~nm}$ and $8 \mathrm{~nm}$ for the InAs barrier and the InAsSb quantum wells respectively. Figure 3(b) shows a high-resolution TEM image of the MQWs demonstrating the formation of 
abrupt interfaces with low Sb segregation. Furthermore, the wells displayed a planar growth mode without any wavy, defect related deformations which can occur for growth on $\mathrm{Si}^{17}$.

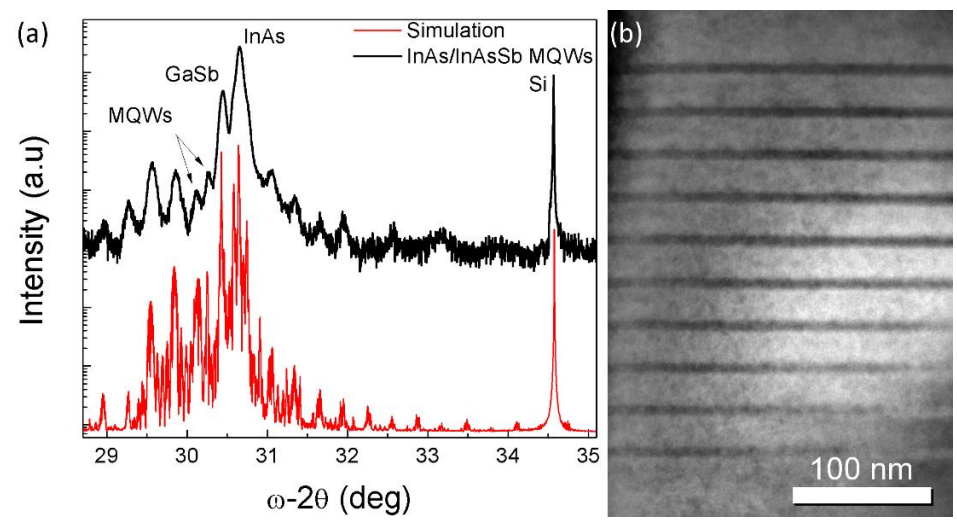

FIG. 3. (a) Experimental and simulated $\omega-2 \theta$ XRD pattern of the MQW sample. (b) Cross section high-resolution TEM image showing the high quality InAs/InAsSb MQW structure.

The optical properties of the InAs/InAsSb MQWs PL sample were then investigated. Power dependent photoluminescence (PL) measurements of the InAs/InAsSb MQW sample were carried out at $15 \mathrm{~K}$. As shown in Figure 4(a), three emission peaks were observed: The first peak, at 351 $\mathrm{meV}$, is in excellent agreement with the theoretically predicted $\mathrm{e}_{1}-\mathrm{hh}_{1}$ transition energy of 355 $\mathrm{meV}$. The second peak, at $374 \mathrm{meV}$, is in agreement with the $381 \mathrm{meV}$ theoretically predicted $\mathrm{e}_{1}$ $\mathrm{hh}_{2}$ transition which is allowed due to the characteristic type-II band alignment of the MQWs. The third peak is attributed to the $400 \mathrm{meV}$ InAs band-to-band transition. At low laser excitation power,the spectra is dominated by the $\mathrm{e}_{1}-\mathrm{h}_{1}$ transition. With increasing laser excitation power, the relative intensity of $e_{1}-h_{2}$ increases. The inset of Figure 4(a) shows the integrated PL intensity of the $\mathrm{e}_{1}-\mathrm{hh}_{1}$ versus the laser excitation power. The data were fitted using equation ${ }^{27}$ :

$$
\mathrm{I}_{\mathrm{PL}}=\mathrm{aP}^{\beta}
$$

where IPL is the integrated intensity, $P$ is the laser excitation power, $\mathrm{a}$ is a constant and $\beta$ is the gradient of the slope related to the dominant recombination process. A gradient close to $1 / 2,1$ or $3 / 2$ indicates the dominance of Shockley-Read-Hall ( $\mathrm{SRH}$ ), radiative or Auger recombination respectively. The gradient, $\beta$, was found to be $0.98 \pm 0.02$, indicating that radiative recombination 
is the dominant recombination process within the wells. This demonstrates the excellent crystalline quality of the sample, with any remaining threading dislocations having only a negligible effect on the optical properties. This could be attributed to the position of the localized defect states above the conduction band for a Ga-free type-II MQWs providing a defect-tolerant structure ${ }^{7}$.
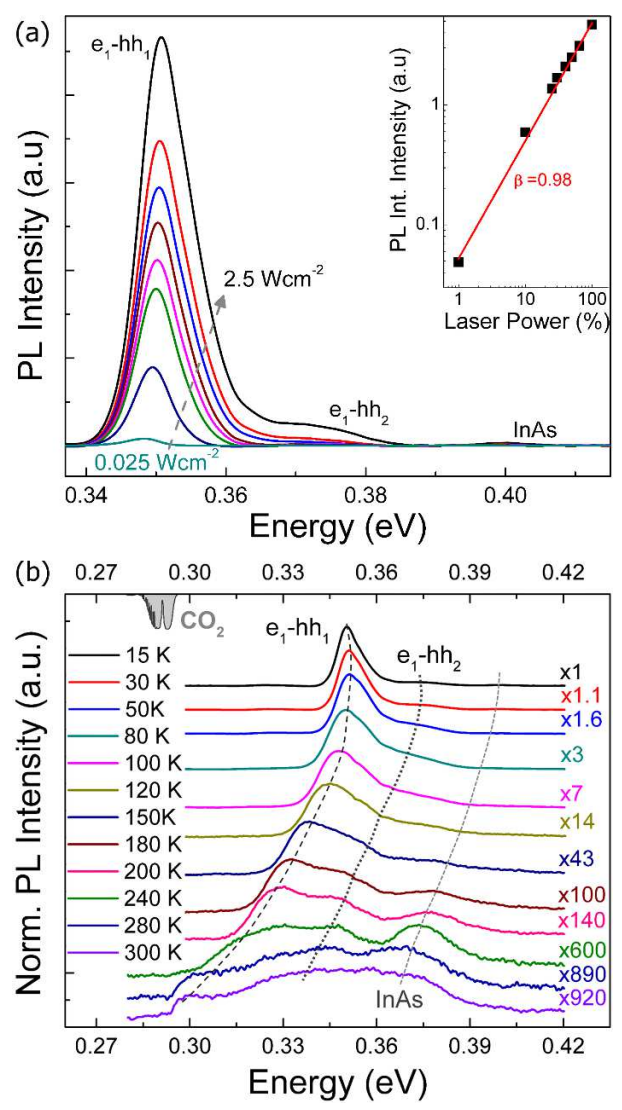

FIG. 4. (a) PL spectra measured for the InAs/InAsSb MQWs at $15 \mathrm{~K}$ for different laser excitation powers. The inset shows the integrated PL intensity dependence on laser excitation power. (b) Temperature dependent PL spectra measured using maximum laser excitation power. The numbers on the right of the spectra show the relative intensities. The lines are guides to the eye indicating the shift of the peaks. 
Figure 4(b) shows the temperature dependent photoluminescence spectra obtained for the MQWs demonstrating strong emission at elevated temperatures. With increasing temperature up to $300 \mathrm{~K}$ all peaks show a shift towards lower energies, with the edge of $\mathrm{e}_{1}-\mathrm{hh}_{1}$ transition peak becoming coincident with the $\mathrm{CO}_{2}$ atmospheric absorption wavelengths. Atmospheric absorption occurred within the optical path of the external light beam between the cryostat, where the sample was placed, and the FTIR detector. The $\mathrm{e}_{1}-\mathrm{hh}_{2}$ transition peak increases in intensity for temperatures higher than $200 \mathrm{~K}$ due to the thermal excitation of holes from the first heavy hole to the second heavy hole band as a result of the small, $26 \mathrm{meV}$, heavy hole band splitting. Furthermore, for temperatures higher than $180 \mathrm{~K}$ the InAs peak becomes significantly stronger relative to the $\mathrm{QW}$ peaks as a result of thermalisation of holes out of the MQWs due to the relatively small confinement energy of $81 \mathrm{meV}$. This could be improved by increasing the Sb content inside the QW.

In summary, a MIR InAs/InAsSb MQW structure, which was heteroepitaxially grown on silicon using an InAs-based buffer structure, exhibited strong photoluminescence signal at room temperature. TEM images revealed the strong threading dislocation filtering effect of the InAs/GaSb interface. A series of InAs-based dislocation filter superlattices were used to confine any remaining threading dislocations to the underlying structure. Surface characterization of the sample revealed a smooth surface with a surface dislocation density about an order of magnitude lower than the GaSb buffer layer surface. Finally, the InAs/InAsSb MQWs, which were grown on top of the InAs-based DFSL buffer, exhibited a strong photoluminescence signal covering the spectral range from approximately 3.5 to $4.2 \mu \mathrm{m}$. At low temperatures, the spectra were dominated by emission from the $\mathrm{e}_{1}-\mathrm{hh}_{1}$ transition. With increasing temperature above $200 \mathrm{~K}$, the $\mathrm{e}_{1}-\mathrm{hh}_{2}$ transition becomes dominant due to the thermal excitation of holes from the first to the second heavy hole states. These results demonstrate that InAs/InAsSb MQW emitters can be integrated onto Si using InAs-based DFSLs and GaSb buffer layers, which is an important step in the development of high efficiency, cost-effective MIR light sources for sensor systems.

The authors would like to thank the EPSRC [grant number EP/N018605/1], the Royal Academy of the Engineering [grant number 10216/114], the Joy Welch Educational Charitable Trust and the Lancaster University Research Committee for the financial support for this work. The underlying data in this paper is available from https//.... 
${ }^{1}$ A. H. Atabaki, S. Moazeni, F. Pavanello, H. Gevorgyan, J. Notaros, L. Alloatti, M. T. Wade, C. Sun, S. A. Kruger, H. Meng, K. Al Qubaisi, I. Wang, B. Zhang, A. Khilo, C. V. Baiocco, M. A. Popović, V. M. Stojanović, R. J. Ram, Nature 556, 349-354 (2018).

${ }^{2}$ S. A. Miller, M. Yu, X. Ji, A. G. Griffith, J. Cardenas, A. L. Gaeta, M. Lipson, Optica 4, 7, 707-712 (2017).

${ }^{3}$ A. Spott, E. J. Stanton, A. Torres, M. L. Davenport, C. L. Canedy, I. Vurgaftman, M. Kim, C. S. Kim, C. D. Merritt, W. W. Bewley, J. R. Meyer, J. E. Bowers, Optica 5, 8, 996-1005 (2018).

${ }^{4}$ A. Spott, E. J. Stanton, N. Volet, J. D. Peters, J. R. Meyer, J. E. Bowers, IEEE J. Sel. Top. Quantum Electron. 23, 6 (2017).

${ }^{5}$ S. Kasap, P. Capper, Handbook of Electronic and Photonic Materials (Springer, Cham, 2017) pp. 725-741.

${ }^{6}$ A. M. Hoang, G. Chen, R. Chevallier, A. Haddadi, M. Razeghi, Appl. Phys. Lett. 104, 251105 (2014).

${ }^{7}$ A. D. Prins, M. K. Lewis, Z. L. Bushell, S. J. Sweeney, S. Liu, Y.-H. Zhang, Appl. Phys. Lett. $106,17,171111(2015)$

${ }^{8}$ C. H. Choi, L. Hultman, R. Ai, S. A. Barnett, Appl. Phys. Lett. 57, 27, 2931 (1990).

${ }^{9}$ R. Alcottea, M. Martina, J. Moeyaerta, P. Gergaude, S. Davida, T. Cerbaa, F. Bassania, F. Ducroquet, Y. Bogumilowicze, T. Barona, Thin Solid Films 645,119-123 (2018).

${ }^{10}$ W. Dobbelaere, J. De Boeck, P. Heremans, R. Mertens, G. Borghs, W. Luyten, J. Van Landuyt, Appl. Phys. Lett. 60, 868 (1992).

${ }^{11}$ R. D. Grober, H. D. Drew, JenInn Chyi, S. Kalem, H. Morkoç, J. Appl. Phys. 65, 4079 (1989).

${ }^{12}$ Ş. Kalem, J. Chyi, C. W. Litton, H. Morkoç, S. C. Kan, A. Yariv, Applied Physics Letters 53, 562 (1988).

${ }^{13}$ W. K. Loke, K. H. Tan, S. Wicaksono, S. F. Yoon, MRS Communications 8, 3 (2018).

${ }^{14}$ L. W. Khai, T. K. Hua, L. Daosheng, S. Wicaksono, Y. S. Fatt, IEEE Photonics Technology Letters, 28, 15, 1653-1656 (2016).

15 Q. Durlin, J.P. Perez, L. Cerutti, J.B. Rodriguez, T. Cerba, T. Baron, E. Tournié, P. Christol, Inf. Phys. Tech. 96, 39-43 (2019).

${ }^{16}$ Z. Loghmari, J.-B. Rodriguez, A. N. Baranov, M. Rio-Calvo, L. Cerutti, A. Meguekam, M. Bahriz, R. Teissier, E. Tournié, APL Photonics 5, 041302 (2020). 
${ }^{17}$ H. Nguyen-Van, A. N. Baranov, Z. Loghmari, L. Cerutti, J. -B. Rodriguez, J. Tournet, G. Narcy, G. Boissier, G. Patriarche, M. Bahriz, E. Tournié, Sci. Rep. 8, 7206 (2018).

${ }^{18}$ E. Delli, V. Letka, P. D. Hodgson, E. Repiso, J. P. Hayton, A. P. Craig, Q. Lu, R. Beanland, A. Krier, A. R. J. Marshall, P. J. Carrington, ACS Photonics 6, 538-544 (2019).

${ }^{19}$ E. Delli, P. D. Hodgson, E. Repiso, A. P. Craig, J. P. Hayton, Q. Lu, A. R. J. Marshall, A.

Krier, P. J. Carrington, IEEE Photonics 11, 3, 2200608 (2019).

${ }^{20}$ P. J. Carrington, Q. Zhuang, M. Yin, A. Krier, Semicond. Sci. Technol. 24, 075001, 2009.

${ }^{21}$ See https://www.nextnano.de/index.php for more information about Nextnano

${ }^{22}$ See https://iippeters.github.io/Strainpp/ for further information regarding Strain++.

${ }^{23}$ T. Ward, A. M. Sánchez, M. Tang, J. Wu, H. Liu, D. J. Dunstan, R. Beanland, J. Appl. Phys. $116,063508,(2014)$.

${ }^{24}$ S. Okumura, S. Tomabechi, R. Suzuki, K. Tsunoda, J.-I. Kon, H. Nishino, Jap. J. Appl. Phys. 57, 115502 (2018).

${ }^{25}$ F. Ernst, P. Pirouz, J. Mater. Res. 4, 4, 834-842 (1989).

${ }^{26}$ W. K. Loke, K. H. Tan, S. Wicaksono, S. F. Yoon, MRS Communications 8, 3, 1085-1091 (2018).

${ }^{27}$ J. -B. Wang, D. Ding, S. R. Johnson, S. -Q. Yu, Y. -H. Zhang, Phys. Status Solidi B 244, 8, 2740-2751 (2007).

Words: 3246 
(a)

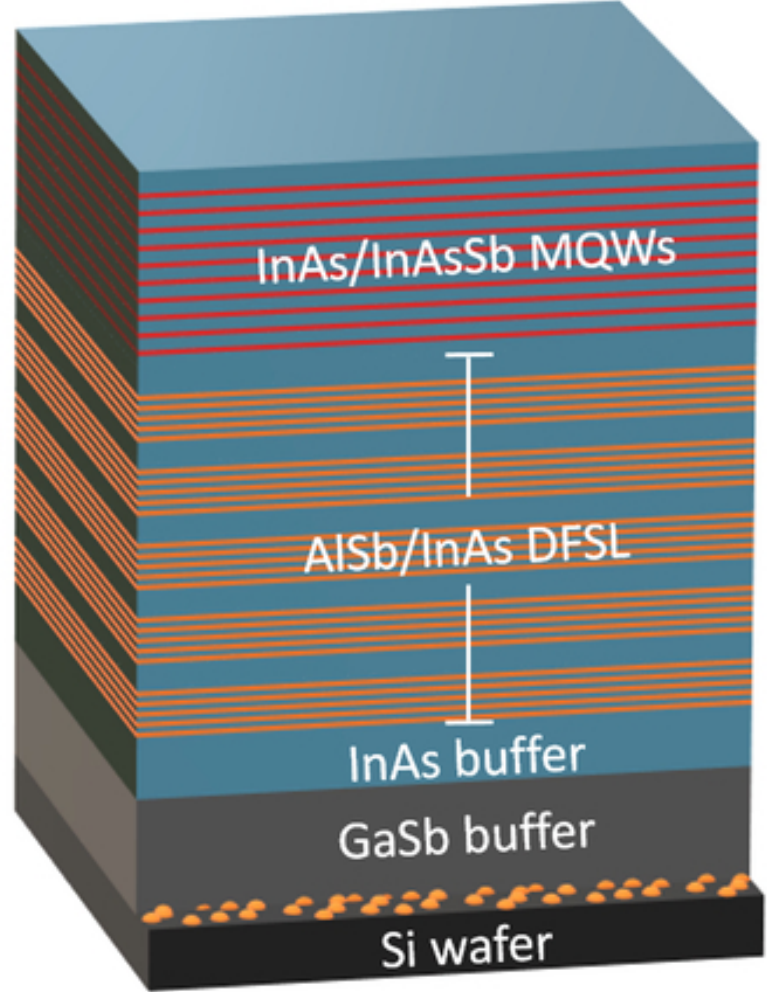

(b)

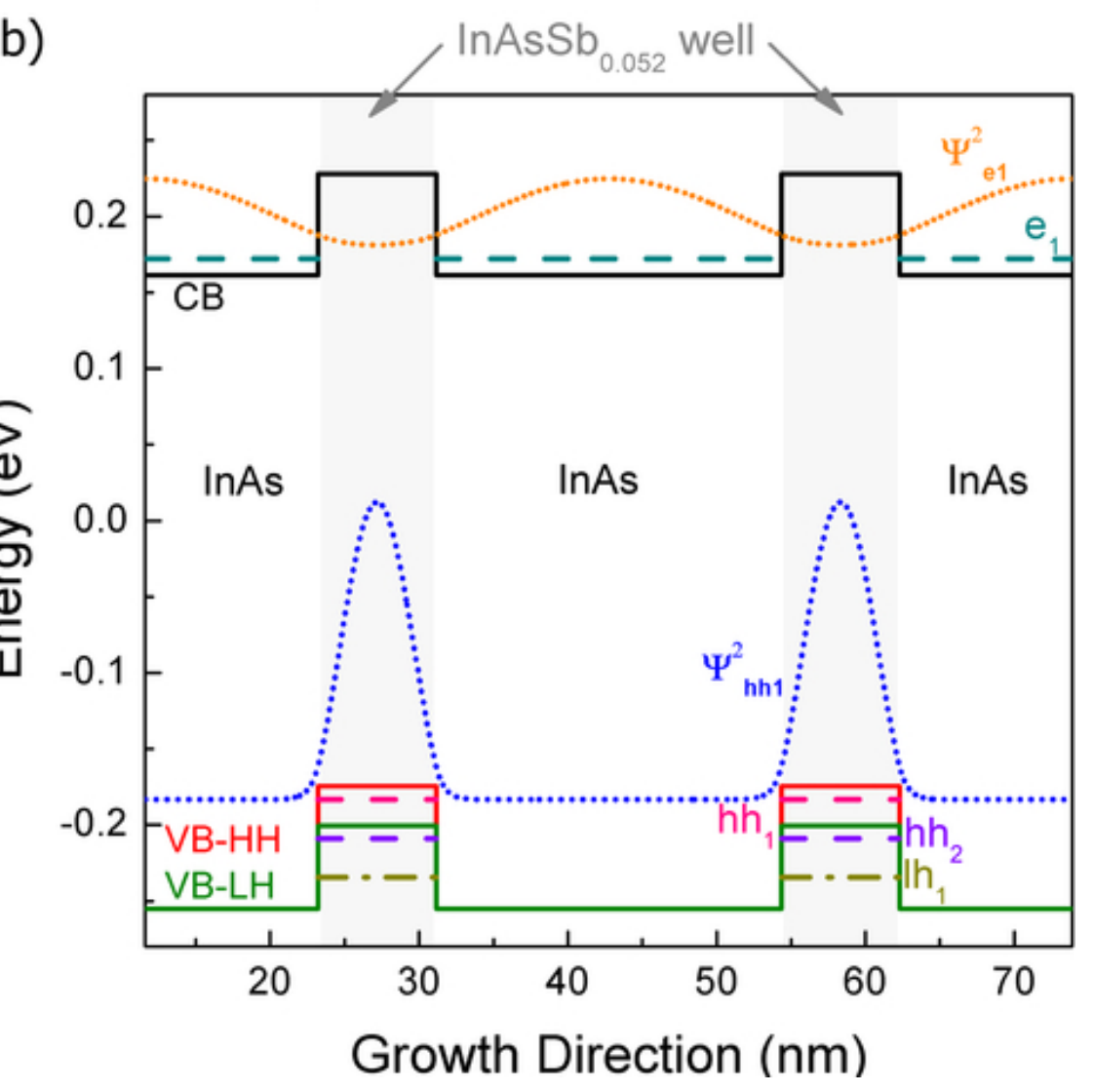




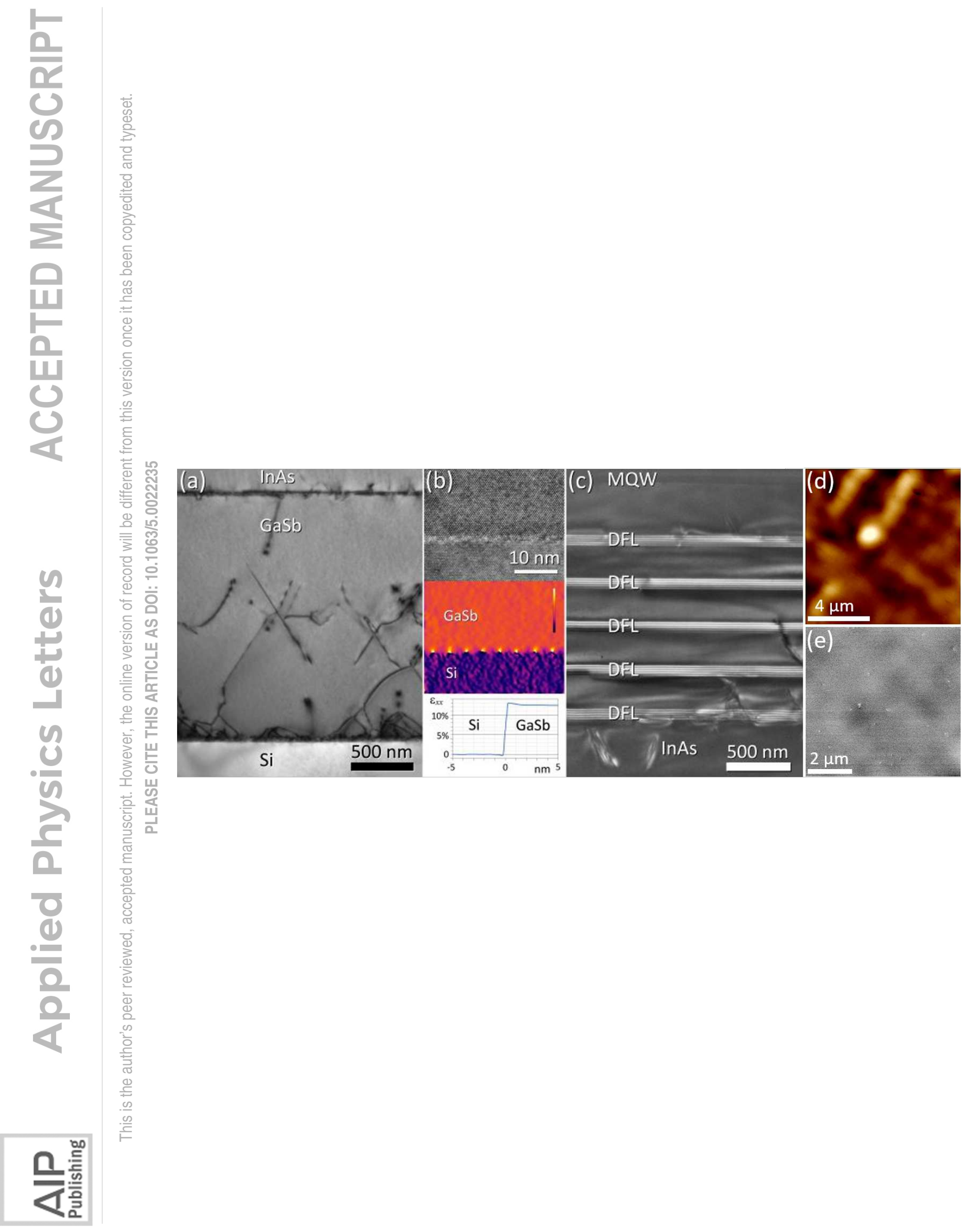



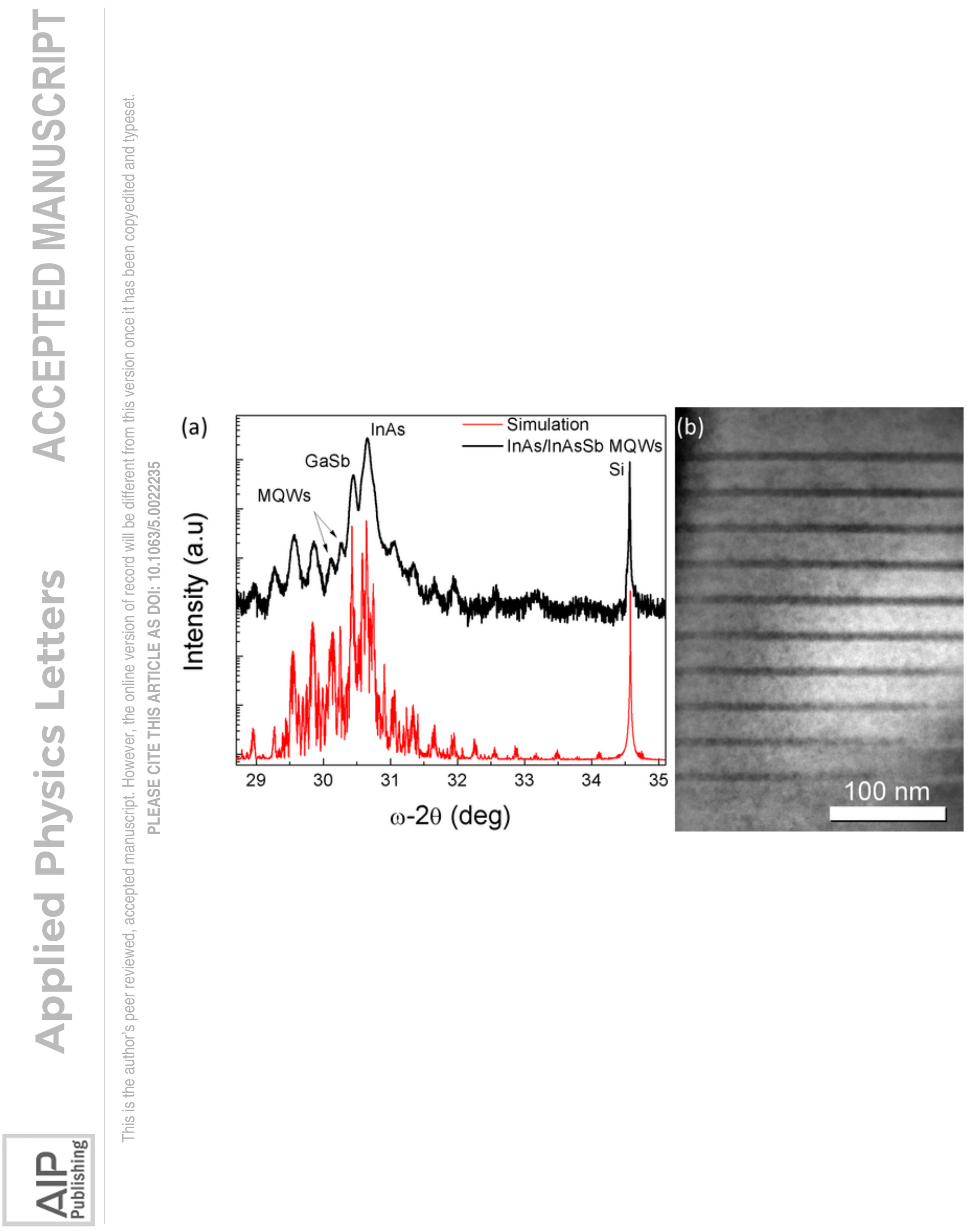

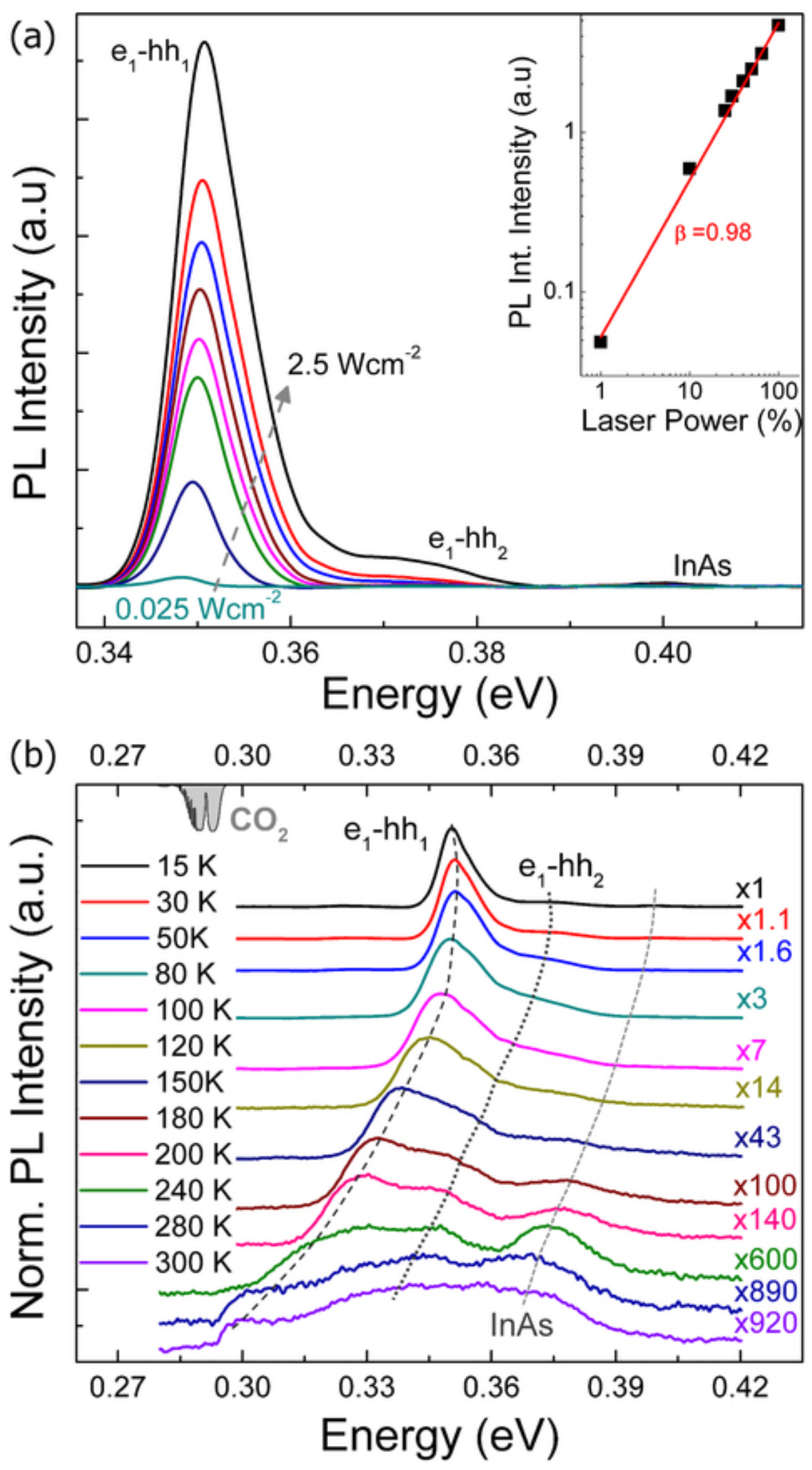